\title{
Effects of HuR downregulation on anaplastic thyroid cancer cells
}

\author{
LORENZO ALLEGRI ${ }^{1}$, CATIA MIO $^{1}$, DIEGO RUSSO $^{2}$, SEBASTIANO FILETTI $^{3}$ and FEDERICA BALDAN $^{3}$ \\ ${ }^{1}$ Department of Medical and Biological Sciences, University of Udine, I-33100 Udine; \\ ${ }^{2}$ Department of Health Sciences, 'Magna Graecia' University of Catanzaro, I-88100 Catanzaro; \\ ${ }^{3}$ Department of Internal Medicine and Medical Specialties, 'Sapienza' University of Rome, I-00161 Rome, Italy
}

Received May 29, 2017; Accepted September 1, 2017

DOI: $10.3892 / 01.2017 .7289$

\begin{abstract}
Anaplastic thyroid cancer (ATC) constitutes one of the most aggressive types of human solid cancer, and is characterized by the absence of thyroid differentiation features and a marked degree of invasiveness. We have previously demonstrated that the RNA-binding protein $\mathrm{Hu}$ antigen $\mathrm{R}(\mathrm{HuR})$ is overexpressed in thyroid carcinoma; thus, the biological role of this RNA-binding protein was investigated in the present study using the ATC cell lines SW1736 and 8505C. In both cell lines, HuR protein levels were higher compared with in the non-tumorigenic thyroid cell line Nthy-ori-3.1. HuR silencing by RNA interference in both ATC cell lines decreased cell viability, increased apoptosis rates and reduced the capability to form colonies in soft agar. Thus, HuR plays an important role in the proliferation and aggressiveness of ATC cells. The histone deacetylase inhibitor suberoylanilide hydroxamic acid (SAHA) was able to reduce the viability of ATC cells. The results demonstrated that SAHA was able to decrease HuR expression in SW1736 and 8505C cells. Furthermore, since it is known that the transcription factor nuclear factor (NF)- $\kappa B$ modulates HuR expression, whether SAHA affects the nuclear (active) fraction of NF- $\kappa$ B in ATC cells was investigated. The data suggested that SAHA decreases ATC cell viability by reducing the active form of $\mathrm{NF}-\kappa \mathrm{B}$, which, in turn, modulates HuR expression.
\end{abstract}

\section{Introduction}

In the last years, it was attested that the most important eukaryotic mechanisms of gene expression regulation occurs at post-transcriptional level (1). These mechanisms are altered in several diseases, including cancer, and this could be due to an aberrant expression and activity of RNA-binding proteins (RBPs) $(2,3)$. These proteins are key regulators of

Correspondence to: Dr Federica Baldan, Department of Internal Medicine and Medical Specialties, 'Sapienza' University of Rome, Viale del Policlinico 155, I-00161 Rome, Italy

E-mail: federica.baldan12@gmail.com

Key words: HuR, RNA-binding proteins, anaplastic thyroid cancer, SAHA, siRNA post-transcriptional gene expression and among them, one the most known RBP to be implicated in tumorigenesis is $\mathrm{Hu}$ antigen $\mathrm{R}$ (HuR) (4-6).

$\mathrm{HuR}$ is the ubiquitously expressed member of the $\mathrm{Hu}$ family and it is involved in regulation of mRNA stability and translation. HuR is located into the nucleus and, in response to stimuli, can shuttle to the cytoplasm to allow its mRNA target to be processed. Several studies demonstrated that $\mathrm{HuR}$ is overexpressed and delocalized in the cytoplasm in numerous cancers, including breast cancer, lung adenocarcinoma, ovarian cancer, laryngeal squamous cell cancer and colon cancer $(6,7)$. Moreover, various mRNA of tumorigenesis factors, oncogenes and anti-apoptotic factors have been identified as HuR targets (5). In a previous study, we demonstrated that HuR is overexpressed also in thyroid cancer (8).

Thyroid cancer is the most widespread endocrine malignancy and although it represent only the 1-2\% of all human neoplasms, its incidence is rapidly growing all over the world in the last decades (9). In most cases, thyroid carcinomas derived from follicular cells and could be classified as differentiated carcinomas, that include follicular thyroid cancer (FTC) and papillary thyroid cancer (PTC), and undifferentiated carcinomas, also named anaplastic thyroid cancer (ATC) (10).

ATC constitute one of the most aggressive and lethal human solid tumor and is characterized by an absence of thyroid differentiation features, a marked degree of invasiveness and extensive necrosis (11). ATC patients have a median survival of 5 months and less than $20 \%$ survive 12 month. Nowadays there are not effective therapies for ATC, since surgery, traditional chemotherapies and radiation therapies are mostly ineffective and are not able to improve the overall survival $(10,11)$. Therefore, innovative approaches for ATC treatment are needed.

\section{Materials and methods}

Cell lines. Nthy-ori-3.1, derived from normal thyroid follicular epithelial cells and immortalized by the SV40 large T gene, SW1736 and 8505C cell lines, from ATC, were grown in RPMI1640 medium (Euro Clone, Milan, Italy) supplemented with $10 \%$ fetal bovine serum (Gibco Invitrogen, Milan, Italy), $2 \mathrm{mM}$ L-glutamine (Euro Clone) and $50 \mathrm{mg} / \mathrm{ml}$ gentamicin (Gibco Invitrogen). Cultured cells were treated with either vehicle (DMSO; Sigma-Aldrich; Merck KGaA, Darmstadt, Germany) or suberoylanilide hydroxamic acid (SAHA) 
(Cayman Chemical, Ann Arbor, MI, USA). Cells were grown in a humidified incubator $\left(5 \% \mathrm{CO}_{2}\right.$ in air at $\left.37^{\circ} \mathrm{C}\right)$ (Eppendorf AG, Hamburg, Germany). All cell lines have been validated by short tandem repeat and tested for being mycoplasma-free.

Protein extraction and western blot. Total protein extraction was performed harvesting Nthy-ori-3.1, SW1736, and 8505C cells by scraping and lysing cells with total lysis buffer (Tris $\mathrm{HCl} 50 \mathrm{mM}$ pH8, NaCl 120 mM, EDTA 5 mM, Triton 1\%, NP40 1\%, DTT $1 \mathrm{mM}$ ), supplemented with phenyl-methylsulphonyl fluoride and protease inhibitors. Lysates, then, were centrifuged at $13,000 \mathrm{x} \mathrm{g}$ for $10 \mathrm{~min}$ at $4^{\circ} \mathrm{C}$ and supernatants were quantified by Bradford assay.

For western blot analysis, proteins were electrophoresed either on $7.5,10$ or $12 \%$ SDS-PAGE and then transferred to nitrocellulose membranes (GE Healthcare, Little Chalfont, UK), saturated with 5\% non-fat dry milk in PBS $/ 0.1 \%$ Tween-20. The membranes were then incubated overnight with rabbit polyclonal anti-HuR antibody 1:500 (EMD Millipore, Billerica, MA, USA), rabbit anti-PARP antibody 1:400 (Abcam, Cambridge, UK), rabbit anti-nuclear factor (NF) $-\kappa B$ antibody 1: 200 (Santa Cruz Biotechnology, Inc., Dallas, TX, USA), rabbit anti-actin antibody 1:1,000 (Abcam) or rabbit anti-LSD1 antibody 1:1,000 (Abcam). The day after, membranes were incubated with anti-rabbit immunoglobulin coupled to peroxidase 1:4,000 (Sigma-Aldrich) for $2 \mathrm{~h}$. Blots were developed using UVITEC Alliance LD (UVItec Ltd., Cambridge, UK) with the SuperSignal Technology (Thermo Fisher Scientific, Inc., Waltham, MA, USA).

$H u R$ silencing. For transient silencing of endogenous HuR in SW1736 and 8505c cells, TriFECTa RNAi kit (Integrated DNA Technologies Inc., Coralville, IA, USA) was used following manufacturer's instructions. A duplex targeting a site absent in human genome was used as 'universal' negative control. Three different siRNA oligonucleotides (siRNA1, siRNA2 and siRNA3) were transfected at a concentration of $1 \mathrm{nM}$ using DharmaFECT 1 Transfection reagent (Thermo Fisher Scientific, Inc.), according to manufacturer's instructions. The day before transfection, cells were plated in antibiotics-free medium. Cells were harvested $72 \mathrm{~h}$ after transfection and gene-silencing efficiency was evaluated by protein levels analysis.

Cell viability. In order to test cell viability, we applied the methyl thiazolyl tetrazolium assay (MTT) assay. SW1736 and $8505 \mathrm{C}$ cells (3,000 cells/well) were plated onto 96-well plates in $200 \mu \mathrm{lmedium} /$ well and were allowed to attach to the plate for $24 \mathrm{~h} \mathrm{(t0)}$. Plates were then incubated for 24, 48 and $72 \mathrm{~h}$. $4 \mathrm{mg} / \mathrm{ml} \mathrm{MTT} \mathrm{(Sigma-Aldrich)} \mathrm{was} \mathrm{then} \mathrm{added} \mathrm{to} \mathrm{the} \mathrm{cell}$ medium and cells were cultivated for another $4 \mathrm{~h}$ darkened in the incubator. The supernatant was removed, $100 \mu \mathrm{l} /$ well of DMSO (Sigma-Aldrich) were added and the absorbance at $570 \mathrm{~nm}$ was measured. All experiments were run in quadruplicate and cell viability was expressed as a fold-change compared to control.

Soft agar assay. SW1736 and 8505C cells clonogenic activity after HuR silencing was evaluated by soft agar assay.
Briefly, after $72 \mathrm{~h} \mathrm{HuR}$ silencing, cells have been collected and 10,000 cells/plate were suspended in $4 \mathrm{ml}$ of complete medium containing $0.25 \%$ agarose and then seeded to the top of a $1 \%$ agarose complete medium layer in $6 \mathrm{~cm}$ plates. The colonies were counted on an inverted microscope Leica DMI-600B (Leica Microsystems Ltd., Heerbrugg, Switzerland). Data are representative of three independent experiments.

Gene expression assays. $500 \mathrm{ng}$ of total RNA of SW1736 and $8505 \mathrm{C}$ cells, treated with SAHA or vehicle, were reverse transcribed to cDNA using random exaprimers and MMLV reverse transcriptase (Life Technologies; Thermo Fisher Scientific, Inc.). Real-time PCR was performed using Platinum SYBR-Green qPCR supermix (Life Technologies; Thermo Fisher Scientific, Inc.) on the ABI Prism 7300 Sequence Detection Systems (Applied Biosystems). The $\Delta \Delta \mathrm{Cq}$ method, by means of the SDS software (Applied Biosystems; Thermo Fisher Scientific, Inc.), was used to calculate mRNA levels. Oligonucleotide primers were purchased from Sigma-Aldrich and their sequences are available upon request.

Statistical analysis. All data obtained are expressed as means \pm standard deviation, and significances were analyzed with the Student's t-test performed with GraphPAD Software for Science (San Diego, CA, USA).

\section{Results}

Since we had previously demonstrated the in vivo HuR overexpression in thyroid cancer and considering the necessity to identify innovative approaches for ATC treatment, in this study we focused on the expression and the importance of HuR in ATC cells. To this purpose, we evaluated HuR protein levels in a normal (Nthy-ori-3.1) and in two ATC (SW1736 and $8505 \mathrm{C}$ ) cell lines. The Western Blot analysis displayed a significant HuR overexpression in both ATC cells (Fig. 1), confirming data obtained in vivo (8).

In order to evaluate the importance of HuR and its biological effects in ATC cells, we performed an RNA interference assay. In a first set of experiments, we performed the silencing using three different HuR-specific siRNA (1 nM). As shown in Fig. 2A and B, in both SW1736 and 8505C cell lines, siRNA 1 and 2 induces a strong HuR silencing, while siRNA 3 seems to have no effects on the RBP expression. Then, we investigated HuR silencing effects on cell viability, apoptosis and clonogenic ability. In both cell lines, $1 \mathrm{nM}$ siRNA 1 treatments induce a slight (about 20\%), but significative cell viability reduction (Fig. 2C). In order to measure HuR silencing effects on apoptosis, we performed a Western Blot analysis of PARP, a well-known caspase substrate that is specifically cleaved during apoptosis (12). As shown in Fig. 2D, siRNA 1 increases apoptosis phenomena compared to control, in both SW1736 and 8505C cells. Since mRNA of genes involved in cell aggressiveness have been identified among known HuR-targets, we investigated the ability of SW1736 and 8505C to form colonies in soft agar, after HuR-silencing. We detected a $40 \%$ reduction of clonogenic ability after siRNA 1 treatments in both SW1736 and 8505c cells (Fig. 2E). Therefore, all these data indicate that HuR 
A

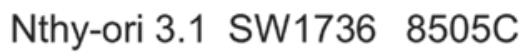

HuR

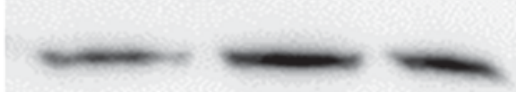

$\beta$-actin

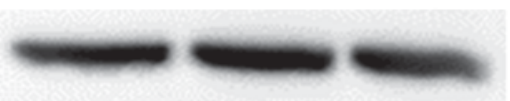

B

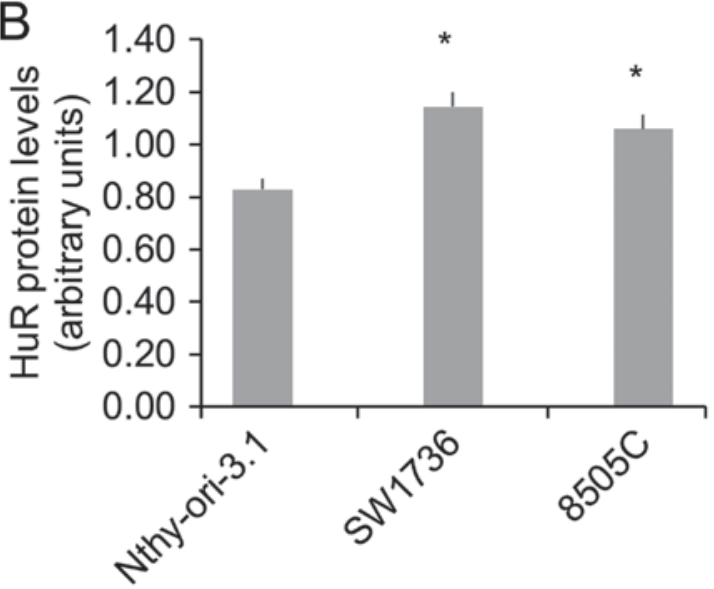

Figure 1. HuR expression in ATC cell lines. (A) Western blot analysis of HuR expression in a non-tumorigenic thyroid cell lines (Nthy-ori-3.1) and in two ATC-derived cell lines (SW1736 and 8505C). (B) Densitometric analysis of HuR protein levels in thyroid cell lines. For each cell line, the results were normalized against $\beta$-actin levels and expressed in arbitrary unit. Results are shown as mean \pm standard deviation. ${ }^{*} \mathrm{P}<0.05$ by Student's t-test.

plays a positive role in cell proliferation and in colonies forming ability in ATC-derived cell lines.

Histone deacetylase (HDAC) inhibitors are an important class of anticancer agents. One of the most known HDAC inhibitor is the SAHA, that is able to induce cell growth arrest and apoptosis in different cancer cell lines (13). In a previously study, we have demonstrated how SAHA $3 \mu \mathrm{M}$ treatment lead to decrement of cell viability in the SW1736 cell line (14). Besides, Zhang et al have demonstrated that the treatment with SAHA induces a reduction of HuR protein expression in mouse epidermal JB6 Cl41 cells (15).

For this reason, after confirming SAHA-related reduction cell viability in $8505 \mathrm{C}$ cells (data not shown), we focused on SAHA effects on HuR protein levels in the two ATC cell lines, in order to determine if SAHA effects in ATC is also due to HuR downregulation. We evaluated, at different time point, whether a $3 \mu \mathrm{M}$ SAHA treatment significantly altered HuR mRNA and protein levels in SW1736 and 8505C cells (Fig. 3). After 48 h SAHA treatment, a reduction about 60 and $50 \%$ of HuR mRNA levels has been detected in SW1736 and 8505C, respectively (Fig. 3A). Thereafter, HuR protein levels have been evaluated after $72 \mathrm{~h}$ SAHA $3 \mu \mathrm{M}$ treatment. As shown in Fig. 3B and C, SAHA induces a strong HuR protein levels decrease in both cell lines.

To define whether SAHA treatment operate directly on HuR transcription or involve other factors implicated in its regulation, we evaluated $\mathrm{NF}-\kappa \mathrm{B}$ protein levels. Kang et al have demonstrated that $\mathrm{NF}-\kappa \mathrm{B}$, binding HuR promoter, directly activates its transcription to promote tumorigenesis (16). NF- $\kappa \mathrm{B}$ is a transcription factor implicated in various aspects of tumor
A
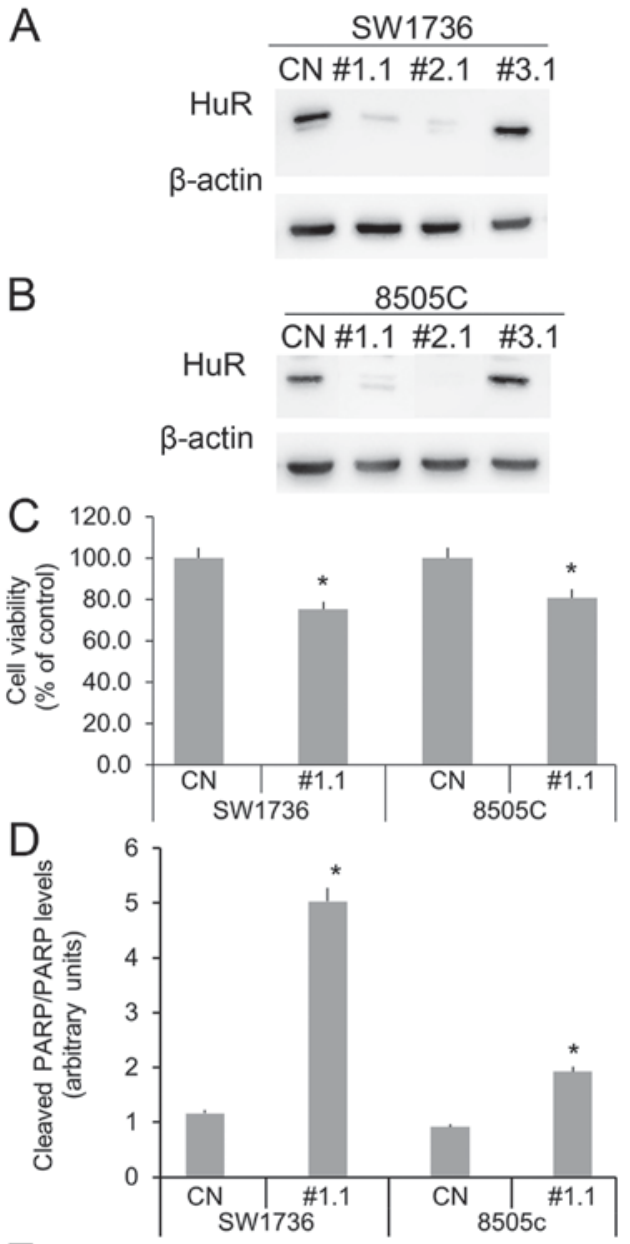

E

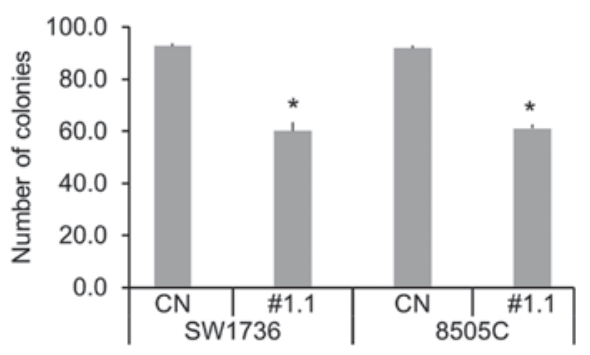

Figure 2. Biological effects of HuR silencing in ATC cells. (A) SW1736 and (B) $8505 \mathrm{C}$ cells were transfected with non-targeting siRNA (CN, negative control) or three different siRNA (1, 2 and 3) sequence specific to HuR $(1 \mathrm{nM})$ and collected after $72 \mathrm{~h}$ treatment. HuR protein levels were analyzed by western blot analysis, as described in Materials and methods section. For each cell line, the results were normalized against $\beta$-actin levels and expressed in arbitrary unit. (C) SW1736 and 8505C cells were transfected to either siRNA1 (\#1.1) or CN for $72 \mathrm{~h}$ and cell viability was analyzed by MTT assay. (D) Densitometric analysis of cleaved PARP fraction levels obtained with western blot assay in SW1736 and 8505C cells transfected to either siRNA1 (\#1.1) or CN for $72 \mathrm{~h}$. (E) Histogram representing the number of colonies per cell line evaluated by colony formation assay of SW1736 and $8505 \mathrm{C}$ transfected to either siRNA1 (\#1.1) or $\mathrm{CN}$ for $72 \mathrm{~h}$. Results are shown as mean \pm standard deviation. " $\mathrm{P}<0.05$ by Student's t-test.

biology, such as cell proliferation, survival, angiogenesis, invasion, metastasis and drug resistance. Inactivated $\mathrm{NF}-\kappa \mathrm{B}$ localized in the cytoplasm and complexed with the inhibitory protein $\mathrm{I} \kappa \mathrm{B} \alpha$ while activated $\mathrm{NF}-\kappa \mathrm{B}$ is translocated into the nucleus where it binds its DNA target sequences $(17,18)$.

In order to evaluate activated NF- $\kappa \mathrm{B}$ levels, we performed a Western Blot analysis on ATC cells nuclear fraction, treated or not with SAHA. Data obtained show that SAHA 


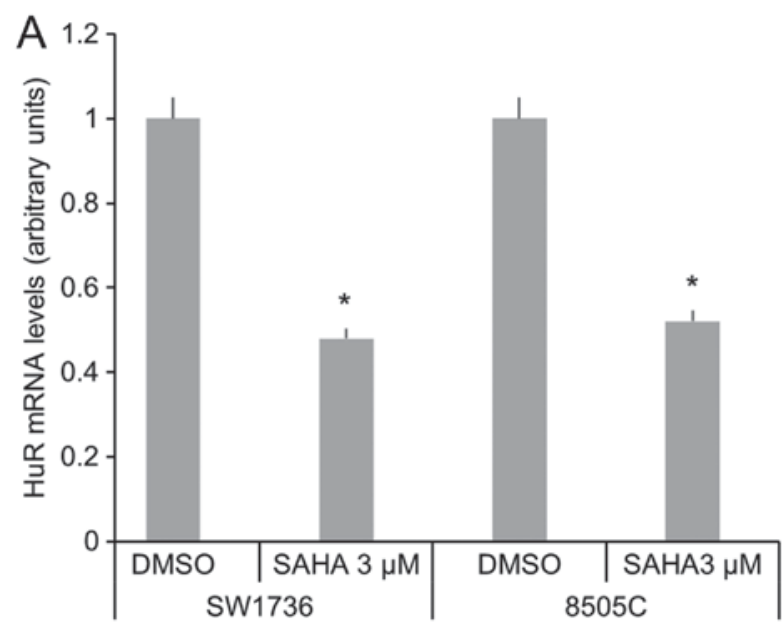

B
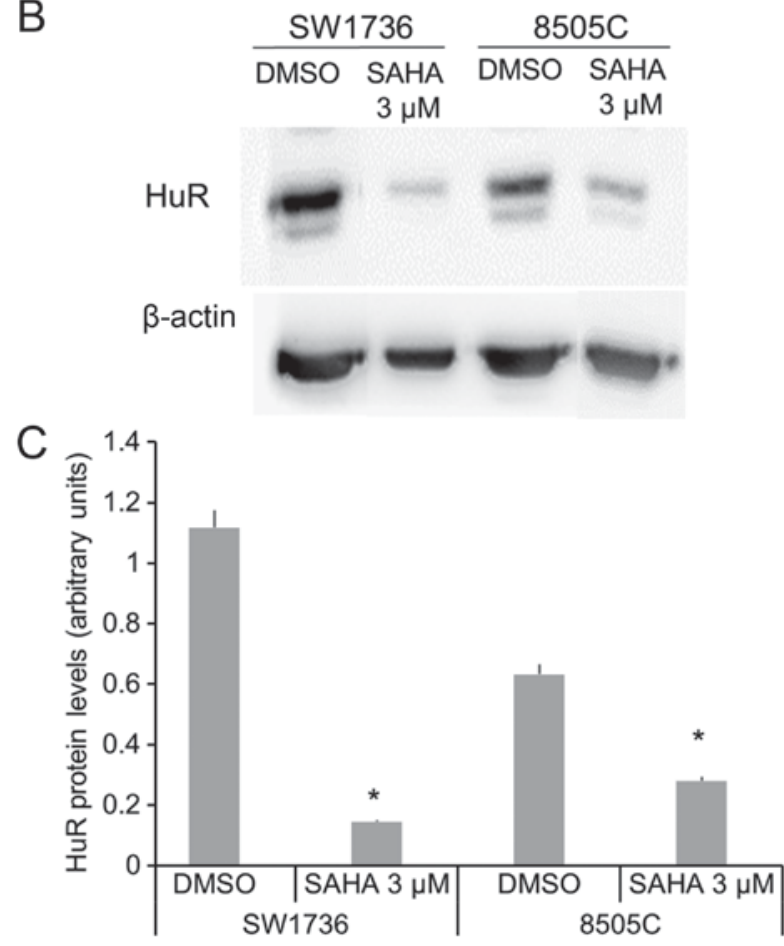

Figure 3. SAHA effects on HuR expression in ATC cells. (A) Relative expression levels of HuR mRNA after SAHA $3 \mu \mathrm{M}$ or DMSO treatment for $48 \mathrm{~h}$. RNA extraction and qPCR are described in Materials and method section For each cell line, the results were normalized against $\beta$-actin levels and expressed in arbitrary unit, calculated as described in Materials and Methods section. (B) Western blot analysis of HuR expression in SW1736 and 8505C treated with SAHA $3 \mu \mathrm{M}$ or DMSO for $72 \mathrm{~h}$. (C) Densitometric analysis of HuR protein levels in ATC cell lines treated with SAHA or DMSO. For each cell line, the results were normalized against $\beta$-actin levels and expressed in arbitrary unit. Results are shown as mean \pm standard deviation. ${ }^{*} \mathrm{P}<0.05$ by Student's t-test.

administration induces a significative reduction of nuclear $\mathrm{NF}-\kappa \mathrm{B}$ fraction after 24 and $48 \mathrm{~h}$ in both SW1736 and $8505 \mathrm{C}$ cells (Fig. 4). These results demonstrate that SAHA treatment reduces activated $\mathrm{NF}-\kappa \mathrm{B}$ levels in ATC cells.

\section{Discussion}

HuR is a RNA-binding protein that plays a major role in regulating gene expression (19) and that can contribute to tumorigenesis (4). HuR transcription is positively regulated

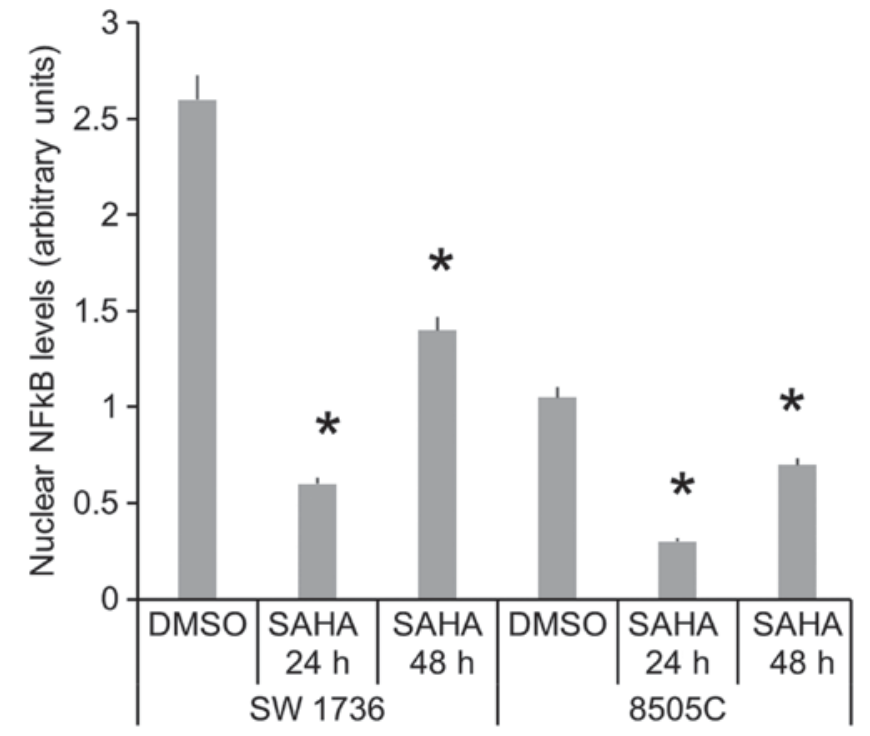

Figure 4. Effects of SAHA treatment on activated NF- $\mathrm{BB}$ levels in ATC cell lines. Densitometric analysis of nuclear NF- $\kappa$ B protein levels in SW1736 and $8505 \mathrm{C}$ cell lines after DMSO or SAHA $3 \mu \mathrm{M}$ administration for 24 or $48 \mathrm{~h}$. For each cell line, the results were normalized against LSD1 expression and expressed in arbitrary unit. Results are shown as mean \pm standard deviation. ${ }^{*} \mathrm{P}<0.05$ by Student's t-test.

by the NF- $\mathrm{BB}$ or by Smad, while the levels of its mRNA and protein are conditioned upon multiple regulation mechanisms $(16,19)$. Several studies indicate that HuR expression and localization are modified in different cancer types, including thyroid cancer $(4,14)$. ATC, although is a rare thyroid cancer histotype, is characterized by a very poor prognosis and a complete absence of differentiation markers (20). Current treatments, based on combination of surgery, chemotherapy and external radiotherapy, are not effective. Therefore, new therapies for ATC are particularly needed.

In order to find new strategies to treat this aggressive thyroid cancer subtype, we investigated HuR expression and its biological effects in two ATC-derived cell lines, SW1736 and $8505 \mathrm{C}$. Therefore, we have demonstrated that HuR is overexpressed in ATC cells and that its silencing determine cell viability reduction due to an increase in apoptosis processes, in both cell lines. Focusing on HuR involvement in cell tumorigenicity, we have established that HuR silencing reduces ATC cells colony forming ability. In this way, we have proved, for the first time, the importance of HuR in ATC cell lines in terms of cell viability, cell death and tumor aggressiveness. Therefore, HuR could be a possible therapeutic target for ATC treatment.

Consequently, in a second experimental setting, we focused on substances able to reduce HuR expression in thyroid cancer. In particular, SAHA, a HDAC inhibitor already FDA-approved for the treatment of several neoplastic diseases (21), proved to induce HuR downregulation in mouse epidermal JB6 Cl41 cells (15). Moreover, in a previous study, we demonstrated SAHA effects on SW1736 cell viability (14), and in this study, these data have been confirmed also in $8505 \mathrm{C}$ cells. Our data shown that SAHA induces a strong HuR mRNA and protein levels reduction, in both ATC cell lines. To better understand the mechanism through which SAHA induces 
HuR downregulation, we investigate the effects of this drug on $\mathrm{NF}-\kappa \mathrm{B}$, which is the major transcription factor of $H u R(16)$. In SW1736 and 8505C cell lines, SAHA treatment determines a decrease of activated NF- $\kappa$ B protein levels. This data corroborate the idea that SAHA effects on HuR expression are not direct, but could be due to its effects of $N F-\kappa B$.

In conclusion, our findings indicate, for the first time, that the RBP HuR plays an important role in ATC tumorigenesis. Moreover, we have demonstrated that the HDAC inhibitor SAHA could be used to obtain a HuR downregulation as consequences of reduction of NF- $\mathrm{NB}$ activation.

\section{Acknowledgements}

This study was supported by Associazione Italiana per la Ricerca sul Cancro (AIRC) (ARIC fellowship Rif. 19481), a grant from Ministero degli Affari Esteri of Italy (Progetti grande rilevanza 2016, project no. PGR02954) and from MIUR (PRIN 2015, project no. 2015HPMLFY-011).

\section{References}

1. Schwanhäusser B, Busse D, Li N, Dittmar G, Schuchhardt J, Wolf J, Chen W and Selbach M: Global quantification of mammalian gene expression control. Nature 473: 337-342, 2011.

2. Kechavarzi B and Janga SC: Dissecting the expression landscape of RNA-binding proteins in human cancers. Genome Biol 15: R14, 2014.

3. Wurth L and Gebauer F: RNA-binding proteins, multifaceted translational regulators in cancer. Biochim Biophys Acta 1849: 881-886, 2015.

4. Abdelmohsen $\mathrm{K}$ and Gorospe M: Posttranscriptional regulation of cancer traits by HuR. Wiley Interdiscip Rev RNA 1: 214-229, 2010.

5. Wurth L: Versatility of RNA-binding proteins in cancer. Comp Funct Genomics 2012: 178525, 2012.

6. Wang J, Guo Y, Chu H, Guan Y, Bi J and Wang B: Multiple functions of the RNA-binding protein HuR in cancer progression, treatment responses and prognosis. Int J Mol Sci 14: 10015-10041, 2013.

7. Govindaraju S and Lee BS: Adaptive and maladaptive expression of the mRNA regulatory protein HuR. World J Biol Chem 4 : 111-118, 2013.
8. Baldan F, Mio C, Allegri L, Conzatti K, Toffoletto B, Puppin C, Radovic S, Vascotto C, Russo D, Di Loreto C and Damante G: Identification of tumorigenesis-related mRNAs associated with RNA-binding protein HuR in thyroid cancer cells. Oncotarget 7: 63388-63407, 2016.

9. Pellegriti G, Frasca F, Regalbuto C, Squatrito S and Vigneri R: Worldwide increasing incidence of thyroid cancer: Update on epidemiology and risk factors. J Cancer Epidemiol 2013: 965212 , 2013.

10. Lebastchi AH and Callender GG: Thyroid cancer. Curr Probl Cancer 38: 48-74, 2014.

11. O'Neill JP and Shaha AR: Anaplastic thyroid cancer. Oral Oncol 49: 702-706, 2013.

12. Chaitanya GV, Alexander JS and Babu PP: PARP-1 cleavage fragments: Signatures of cell-death proteases in neurodegeneration. Cell Commun Signal 8: 31, 2010.

13. Ungerstedt JS, Sowa Y, Xu WS, Shao Y, Dokmanovic M, Perez G, Ngo L, Holmgren A, Jiang X and Marks PA: Role of thioredoxin in the response of normal and transformed cells to histone deacetylase inhibitors. Proc Natl Acad Sci USA 102: 673-678, 2005.

14. Baldan F, Mio C, Allegri L, Puppin C, Russo D, Filetti S and Damante G: Synergy between HDAC and PARP inhibitors on proliferation of a human anaplastic thyroid cancer-derived cell line. Int J Endocrinol 2015: 978371, 2015.

15. Zhang J, Ouyang W, Li J, Zhang D, Yu Y, Wang Y, Li X and Huang C: Suberoylanilide hydroxamic acid (SAHA) inhibits EGF-induced cell transformation via reduction of cyclin D1 mRNA stability. Toxicol Appl Pharmacol 263: 218-224, 2012.

16. Kang M, Ryu B, Lee M, Han J, Lee JH, Ha TK, Byun DS, Chae KS, Lee BH, Chun HS, et al: NF-kappaB activates transcription of the RNA-binding factor HuR, via PI3K-AKT signaling, to promote gastric tumorigenesis. Gastroenterology 135: 2030-2042. e1-3, 2008.

17. Gilmore TD: Introduction to NF-kappaB: Players, pathways, perspectives. Oncogene 25: 6680-6684, 2006.

18. Perkins ND: Integrating cell-signalling pathways with NF-kappaB and IKK function. Nat Rev Mol Cell Biol 8: 49-62, 2007.

19. Srikantan S and Gorospe M: HuR function in disease. Front Biosci (Landmark Ed) 17: 189-205, 2012.

20. Blaxall BC, Dwyer-Nield LD, Bauer AK, Bohlmeyer TJ, Malkinson AM and Port JD: Differential expression and localization of the mRNA binding proteins, AU-rich element mRNA binding protein (AUF1) and $\mathrm{Hu}$ antigen $\mathrm{R}(\mathrm{HuR})$, in neoplastic lung tissue. Mol Carcinog 28: 76-83, 2000.

21. Mann BS, Johnson JR, Cohen MH, Justice R and Pazdur R: FDA approval summary: Vorinostat for treatment of advanced primary cutaneous T-cell lymphoma. Oncologist 12: 1247-1252, 2007. 